\title{
Perforación profunda en el lago de Chalco: reporte técnico
}

Socorro Lozano-García, Erik T. Brown, Beatriz Ortega, Margarita Caballero, Josef Werne,

Peter J. Fawcett, Antje Schwalb, Blas L. Valero-Garcés, Douglas Schnurrenberger, Ryan O'Grady, Mona Stockhecke, Byron Steinman, Enrique Cabral-Cano, Cecilia Caballero, Susana Sosa-Nájera, Ana María Soler, Liseth Pérez, Anders Noren, Amy Myrbo, Matthias Bücker, Nigel Wattrus, Alejandra Arciniega, Thomas Wonik, Sebastian Watt, Dervla Kumar, Carmen Acosta, Ivan Martínez, Rafael Cossio, Troy Ferland, Filiberto Vergara-Huerta

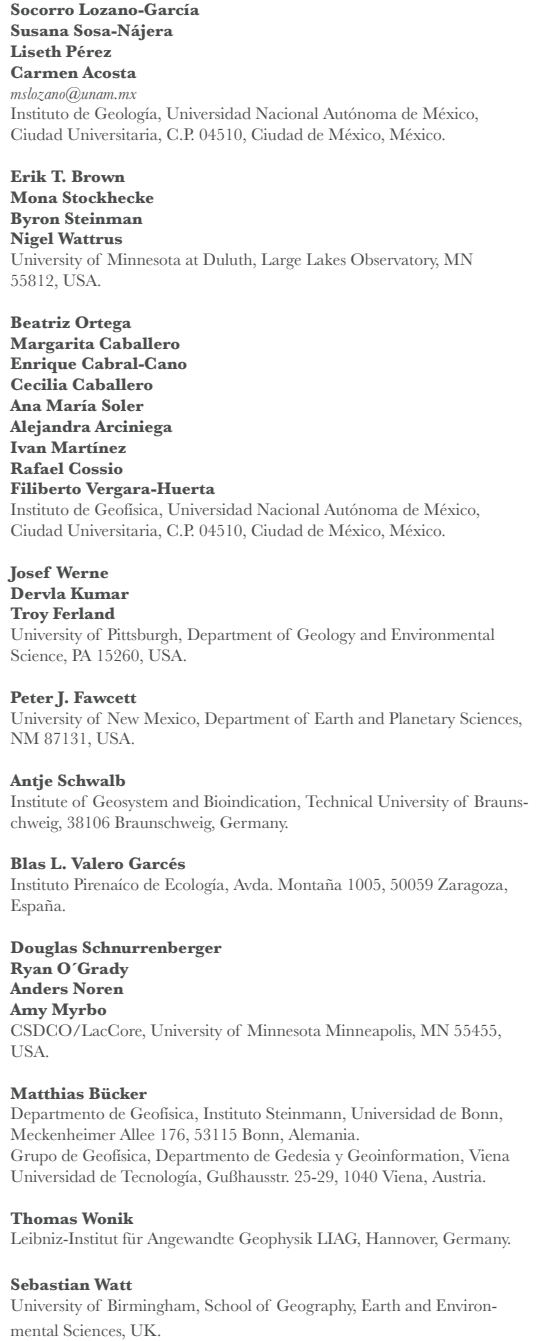

University of Birming
mental Sciences, UK.

BOL. SOG. GEOL. MEX. 2017

VOL. 69 NO. 2

P. $299-311$

Manuscrito recibido: Septiembre 21, 2016. Manuscrito corregido: Noviembre 28, 2016. Manuscrito aceptado: Diciembre 3, 2016.

\section{RESUMEN}

En este artículo se presenta un resumen de las actividades realizadas para la recuperación de la totalidad de la secuencia lacustre del lago de Chalco. Mediante estudios geofísicos se determinó la distribución y espesor de los sedimentos lacustres con base en lo cual se seleccionó el sitio de perforación. Con datos de los espectros $\mathrm{H} / \mathrm{V}$ de sísmica pasiva se hizo un mapa de isofrecuencias que definieron una región con sedimentos lacustres y material volcánico granulado de hasta $300 \mathrm{~m}$ de espesor. El uso de métodos electromagnéticos mostró cambios en la resistividad eléctrica relacionados con variaciones en la composición de la columna sedimentaria; entre $100-120 \mathrm{~m}$ de profundidad hay un primer aumento en la resistividad asociado al incremento de materiales volcaniclásticos, y entre 330 - $400 \mathrm{~m}$ de profundidad un segundo aumento asociado a la presencia de coladas de basalto. Fueron perforados tres pozos con recuperación continua, llegando a profundidades de $420 \mathrm{~m}$ en el pozo A, $310 \mathrm{~m}$ en el B y 520 en el C. Durante el trabajo de perforación se tomaron muestras para el análisis geomicrobiológicos y de metagenómica. Durante el proceso de perforación se recuperó un total de 1152 m de sedimentos con una profundidad máxima de $520 \mathrm{~m}$. El porcentaje de recuperación de la columna sedimentaria varió entre 88 a $92 \%$ en los tres sondeos. Los resultados del análisis de susceptibilidad magnética en las tres secuencias indica que los primeros $260 \mathrm{~m}$ son sedimentos lacustres, entre 260 y $300 \mathrm{~m}$ los sedimentos son más gruesos y debajo de los 300 $\mathrm{m}$ son predominantemente volcaniclásticos. E análisis de la secuencia sedimentaria del lago de Chalco de los últimos 300000 años, permitirá documentar y ampliar el conocimiento acerca de la variabilidad climática de la zona, la historia paleoambiental, la historia del cierre de la cuenca, el desarrollo del sistema lacustre y la recurrencia de la actividad volcánica en la cuenca. Además, el estudio de las propiedades físicas de esta secuencia sedimentaria es importante para la modelación de la propagación de ondas sísmicas y de la estructura de la cuenca, así como para mejorar la capacidad de modelación del proceso de subsidencia de terreno que experimenta esta región.

Palabras clave: Sedimentos lacustres, paleoclima, vulcanismo, Cuenca de México, Chalco.

\section{ABSTRACT}

This paper presents a short description of the coring operations undertaken to recover the full lacustrine sedimentary sequence from Chalco. Geophysical techniques were used to determine the distribution and thickness of the sediments in order to select the drilling site. Resonance frequencies determined from $H / V$ spectral ratios were used to determine an area where lake sediments reached 300 m thickness. Electromagnetic survey showed two changes in electric resistivity which were related to changes in sediment composition, the first from 100 to $120 \mathrm{~m}$, related to an increase in volcanoclastic sediments and the second from 330 to $400 \mathrm{~m}$ related to the presence of a basaltic flows. Three wells were drilled with continuous recovery, reaching depths of $420 \mathrm{~m}$ in well A, 310 in B and 520 in C. Samples for geomicrobiological and metagenomics studies were collected during drilling operations. A total of $1152 \mathrm{~m}$ of core sediments were recovered reaching a maximum depth of $520 \mathrm{~m}$. Recovery percentages were between 88 and $92 \%$ in the three wells. Magnetic susceptibility analyses in the three sequences show that the first $260 \mathrm{~m}$ are mostly lake sediments, between 260 and 300 $m$ sediments are coarser and below $300 \mathrm{~m}$ they are mostly volcaniclastic. Analysis of the sedimentary sequence of Lake Chalco that covers the last $\sim 300000$ years will allow documenting and extending the knowledge of climate variability in area, the paleoenvironmental history, basin closure history, lacustrian system development and volcanic activity recurrence. Studies of the physical properties of this sequence will be important for seismic propagation and basin structure modeling, and also will improve modeling of the subsidence process that this region experiences.

Keyzords: Lacustrian sediments, paleoclimate, volcanism, Basin of Mexico, Chalco. 


\section{Introducción}

La Faja Volcánica Transmexicana, es una provincia activa desde el Mioceno hasta el presente en la que se desarrollaron cuencas intermontanas (e.g. Gómez-Tuena et al., 2005); una de ellas es la cuenca de México que se localiza en su porción centro-este $\left(19^{\circ} 30^{\prime} \mathrm{N}, 99^{\circ} \mathrm{W}, 9600 \mathrm{~km}^{2}, 2240\right.$ msnm) (Fig. 1A). La actividad volcánica asociada al desarrollo del campo volcánico de la Sierra Chichinautzin cerró el drenaje de esta cuenca hace 1.2 Ma (Arce et al., 2013), lo cual favoreció la acumulación de un importante espesor de sedimentos aluviales, fluviales y el desarrollo de un sistema lacustre (Pérez-Cruz, 1988; Campos-Enríquez et al., 1997; Arce et al., 2013). En los sedimentos de este sistema quedó capturado el registro de la historia ambiental de esta región (p.ej. Bradbury, 1989; Lozano-García et al., 1993; Lozano-García y Ortega-Guerrero, 1998).

La subcuenca de Chalco se localiza en el sector sureste de la cuenca de México (Fig. 1B), en donde abarca un área de $1200 \mathrm{~km}^{2}$, de los cuales la planicie tiene una superficie de $120 \mathrm{~km}^{2}$. El régimen hidráulico de Chalco, así como el del resto de la cuenca de México, fue modificado históricamente para evitar inundaciones y mejorar su aprovechamiento agrícola desde épocas prehispánicas, y actualmente continúa transformándose por la expansión de las áreas urbanas y la extracción de agua del subsuelo. Actualmente, sólo existen alrededor de $5 \mathrm{~km}^{2}$ de espejo lacustre que se ha formado por un proceso de subsidencia del suelo, durante los últimos 20 a 25 años (Cabral-Cano et al., 2010), y $\sim 30 \mathrm{~km}^{2}$ de pastizales y campos agrícolas que son terrenos ejidales de los pueblos originarios de la zona (e.g. Tulyehualco, Tláhuac, Mixquic). La región lacustre de Chalco es una de las pocas zonas sin urbanizar dentro de la Zona Metropolitana de la Ciudad de México que tiene una población que supera los 20 millones de habitantes. Es una área que en menos de 20 años pasó de ser predominantemente rural a predominantemente urbana, y en donde actualmente habitan poco más de 300 mil personas (INEGI http:// www.inegi.org.mx)

La zona del antiguo lago de Chalco es un sitio de particular interés, ya que en sus sedimentos lacustres está registrada la historia climática y geológica de la región de los últimos cientos de miles de años. De acuerdo con las tasas de sedimentación estimadas en estudios anteriores (Lozano-Garćía et al., 1993; Caballero y Ortega-Guerrero, 1998; Ortega-Guerrero et al., 2015; Torres-Rodríguez et al., 2015), una secuencia de $300 \mathrm{~m}$ de espesor en esta cuenca podría contener el registro sedimentario y climático de los últimos 300000 años aproximadamente. El estudio de registros paleoambientales de largo alcance temporal en las regiones tropicales es de suma importancia para entender los procesos de cambio climático que han impactado a estas latitudes en diferentes escalas temporales, como por ejemplo los ciclos orbitales (e.g. ciclos de Milankovitch), que controlan la variabilidad climática en escala de centenas y decenas de miles de años y que están asociados con las oscilaciones climáticas glaciales-interglaciales que han caracterizado los últimos $2 \mathrm{Ma}$ de la historia del planeta.

\section{Estudios iniciales}

En los años 1987 - 1989 se llevó a cabo un conjunto de perforaciones (Figura 1C) que permitieron recuperar y analizar los $25 \mathrm{~m}$ superiores de la secuencia sedimentaria (Lozano-García y Ortega-Guerrero, 1994, 1998; Urrutia-Fucugauchi et al., 1994; Caballero y Ortega-Guerrero, 1998; Ortega-Guerrero et al., 2000). Los resultados del análisis paleoambiental de las primeras perforaciones confirmaron que en sus sedimentos está preservado un excelente registro paleoambiental, en el que se identificaron cambios importantes en el sistema lacustre, en la cobertura vegetal de la cuenca, así como evidencias de la actividad volcánica pasada y la ocurrencia de paleo-incendios. Posteriormente, en 2008, fueron perforados los $122 \mathrm{~m}$ superiores de la secuencia lacustre (He- 

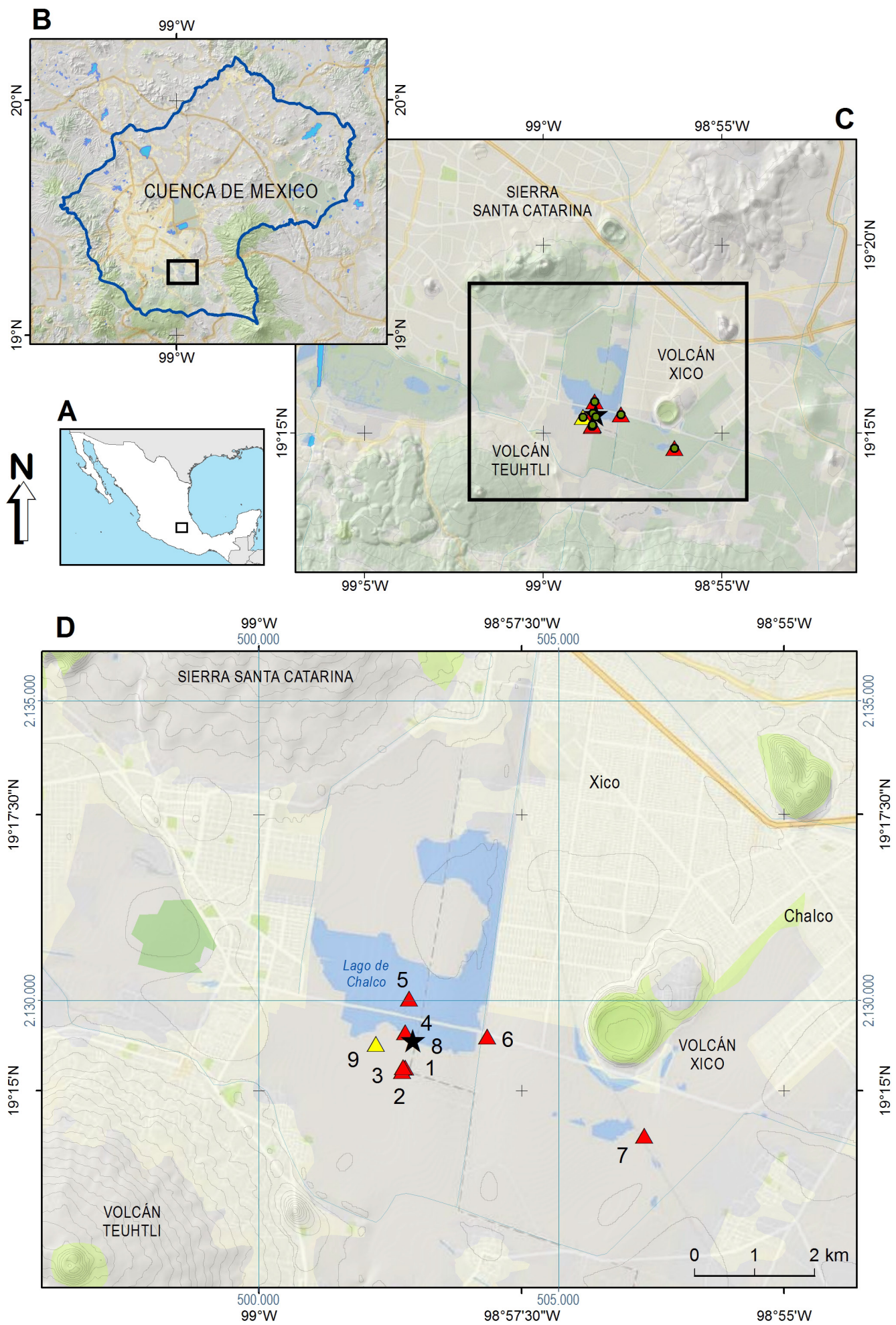

Figura 1 A) Contorno de México señalando la localización de la cuenca de México en el recuadro negro. B) Mapa de la cuenca de México y la zona del lago de Chalco señalada por el recuadro negro. C) Zona del lago de Chalco donde se muestran con triángulos los estudios previos realizados en Chalco (Lozano-García et al., 1993; Caballero y Ortega-Guerrero, 1998; Herrera-Hernández, 2011; Lozano-García et al., 2015. D) Se muestran el sitio de perforación del proyecto Mexidrill con una estrella negra y las perforaciones anteriores con triángulos rojos, el triángulo amarillo indica el área donde se realizó el levantamiento geofísico por sondeo electromagnéticos (Bücker et al., 2017). 
rrera-Hernández, 2011; Ortega-Guerrero et al., 2015; Torres-Rodríguez et al., 2015, Lozano-García et al., 2015) (Figura 1D) en donde se recuperaron sedimentos lacustres en los que dominan facies clásticas (limos y arcillas) con abundantes restos orgánicos (diatomeas, polen, ostrácodos, etc.), intercalados con depósitos volcaniclásticos, siendo los sedimentos lacustres mayormente masivos en los 90 m superiores y laminados de los 90 a 122 m. Finalmente en el 2014 (Figura 1D), se obtuvo el apoyo económico del consorcio "International Scientific Continental Drilling Program" (ICDP, MexiDrill: The Basin of Mexico Drilling Program) y en 2015 de la UNAM (PAPIIT IV00215 "Cambio climático y medio ambiente en la historia del lago de Chalco") y de la National Science Foundation, de Estados Unidos de América, (NSF 1551311 MexiDrill: The Basin of Mexico Drilling Program) que permitieron llevar a cabo la perforación de $521 \mathrm{~m}$ que corresponde a la totalidad de la secuencia sedimentaria lacustre en Chalco, durante los meses de febrero y marzo del 2016.

\section{Objetivo}

Con base en la información recabada a partir de los estudios previos realizados en la zona, se planteó como meta la recuperación de la totalidad de la secuencia de sedimentos lacustres de la cuenca de Chalco a través de una perforación altamente especializada de recuperación continua de núcleos que alcanzara los depósitos aluviales, fluviales y volcaniclásticos que le subyacen. El estudio de la secuencia sedimentaria permitirá documentar y ampliar el conocimiento acerca de la variabilidad climática de la zona, la historia del cierre de la cuenca, el desarrollo del sistema lacustre, y la actividad volcánica. En particular, este estudio es relevante para las personas que viven en esta región ya que se podrá ampliar el conocimiento sobre la recurrencia e intensidad de la actividad volcánica explosiva a través del estudio de las capas de tefras presentes en la secuencia estratigráfica, que actualmente se extiende solo a los últimos 30000 años (Siebe et al., 2005; Arana-Salinas et al., 2010; Guilbaud et al., 2015). Además, estudiar las propiedades físicas de esta secuencia sedimentaria es importante para la modelación de la propagación de ondas sísmicas y de la estructura de la cuenca, así como para mejorar la capacidad de modelación del proceso de subsidencia del terreno que experimenta esta región (Cabral-Cano et al., 2010).

\section{Estimación del espesor de sedimentos en la subcuenca de Chalco mediante técnicas geofísicas}

Con el fin de estimar los espesores y la arquitectura del paquete sedimentario lacustre en la subcuenca de Chalco y guiar una adecuada selección del sitio de perforación se llevaron a cabo estudios de sísmica activa y pasiva en la zona. Posteriormente, la aplicación de métodos eléctricos permitió caracterizar a las unidades de sedimentos y roca de acuerdo a su densidad, lo que facilitó la selección de la herramienta adecuada para llevar a cabo durante la perforación.

\subsection{TÉGNIGA DE GOGIENTES ESPEGTRALES H/V}

$\mathrm{El}$ análisis de sísmica activa resultó muy limitado debido a la escasa penetración de la señal sísmica, por lo que la selección del sitio se guió únicamente por el levantamiento de sísmica pasiva (vibraciones ambientales), cuya metodología se detalla a continuación.

El trabajo de campo cubrió un área de aproximadamente $8 \mathrm{~km}^{2}$ y consistió en registrar 30 minutos de ruido sísmico ambiental en 68 sitios de la zona de estudio, divididos en dos mallas equiespaciadas a $250 \mathrm{~m}$ y $500 \mathrm{~m}$ (Figura 2). Estos registros se tomaron a una frecuencia de muestreo de $128 \mathrm{~Hz}$ con un sismógrafo triaxial Tromino con ancho de banda de 0.1 - $300 \mathrm{~Hz}$. El conjunto de datos fue procesado con la técnica de cocientes espectrales $\mathrm{H} / \mathrm{V}$ considerando la teoría de campos difusos (Sánchez-Sesma et al., 2011). Para estimar los espesores del depósito vulcano-sedimentario y pro- 


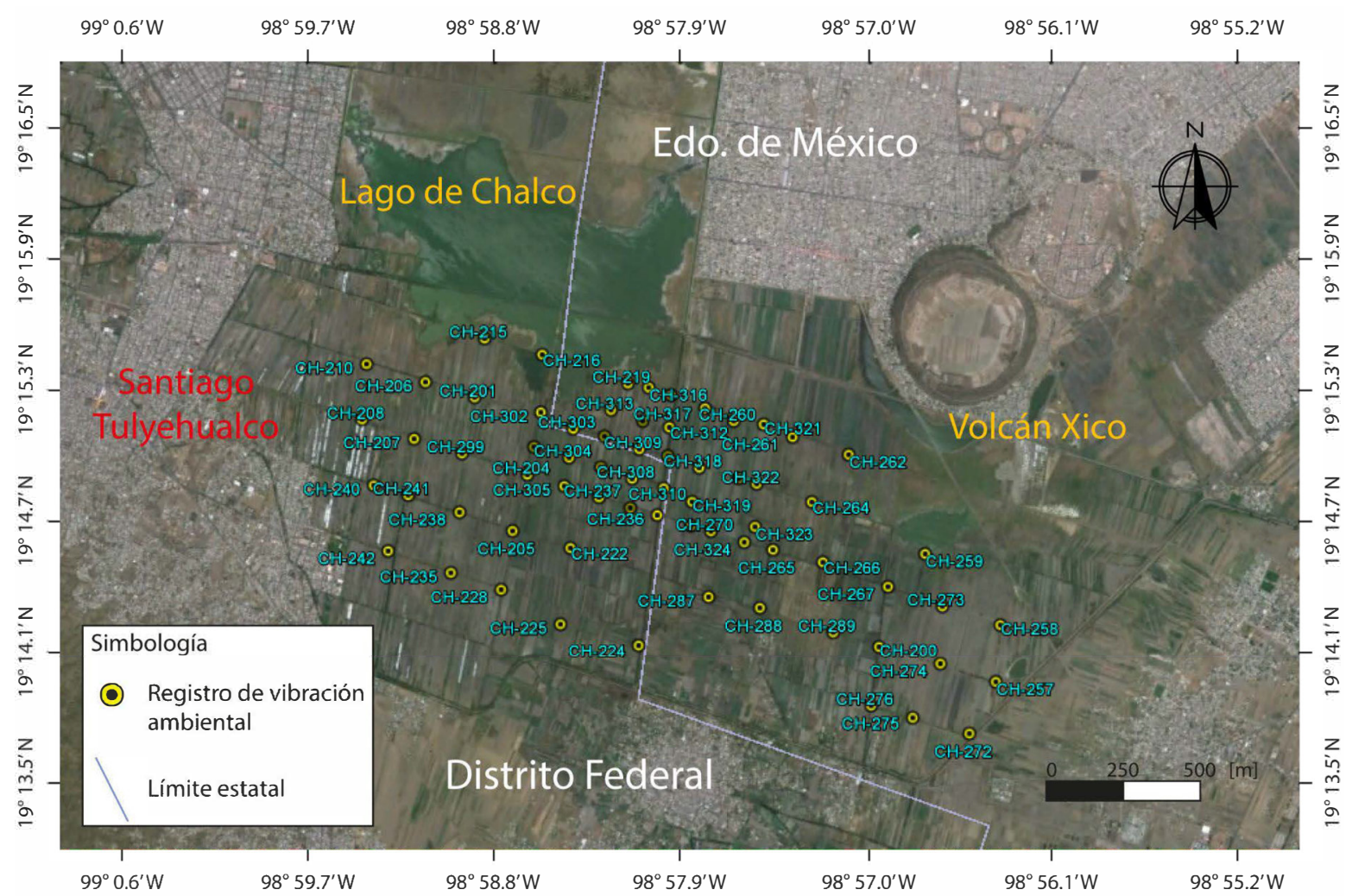

Figura 2 Mapa de localización de los sitios de registro de vibración ambiental (círculos de color amarillo) tomado de Vergara-Huerta, 2015.

poner un modelo de velocidad sísmica de ondas de cortante "S" en el área de estudio, se definieron las frecuencias dominantes de cada sitio de medición. Para esto, se calcularon los cocientes espectrales por sitio tomando ventanas de 40 segundos y un traslape del $30 \%$ a lo largo del registro. El cociente espectral H/V final y su desviación estándar asociada se obtuvieron promediando los cocientes $\mathrm{H} / \mathrm{V}$ de todas las ventanas de tiempo de cada sitio. Las frecuencias dominantes se identificaron de los espectros $\mathrm{H} / \mathrm{V}$, y se elaboró un mapa de isofrecuencias, a parir del cual se obtuvo la distribución de espesores del depósito lacustre (Figura 3).

\subsection{TÉGNIGAS DE SONDEO POR TRANSITORIOS ELEGTROMAGNÉTICOS Y SONDEOS MAGNETOTELÚRICOS}

En febrero de 2016, el Departamento de Sismotectónica y Exploración Geofísica de la CFE, en cooperación con la empresa Geotem Ingeniería
S.A. de G.V., llevó a cabo un levantamiento geofísico en la cercanía al sitio de perforación (Figura $4 \mathrm{~A}$ y $4 \mathrm{~B})$ para caracterizar los contactos geológicos en la zona (Bücker et al., 2017). Esto se logró a través de la aplicación de sondeos por transitorios electromagnéticos (TEM) y sondeos magnetotelúricos (MT). El resultado más importante fue la correlación estrecha entre la resistividad eléctrica obtenida a través de los métodos electromagnéticos (TEM, MT) y las unidades principales de la columna estratigráfica de los sedimentos recuperados en las perforaciones realizadas en el 2008 (Herrera-Hernández, 2011; Torres-Rodríguez et al., 2015). Los modelos de ambos métodos mostraron un primer aumento de la resistividad eléctrica a una profundidad aproximada de 100 a 120 $\mathrm{m}$, el que representa un cambio de composición del sedimento que puede estar relacionado al incremento de sedimentos volcaniclásticos y/o a la presencia de sedimentos lacustres finamente la- 


\section{Mapa de espesores}

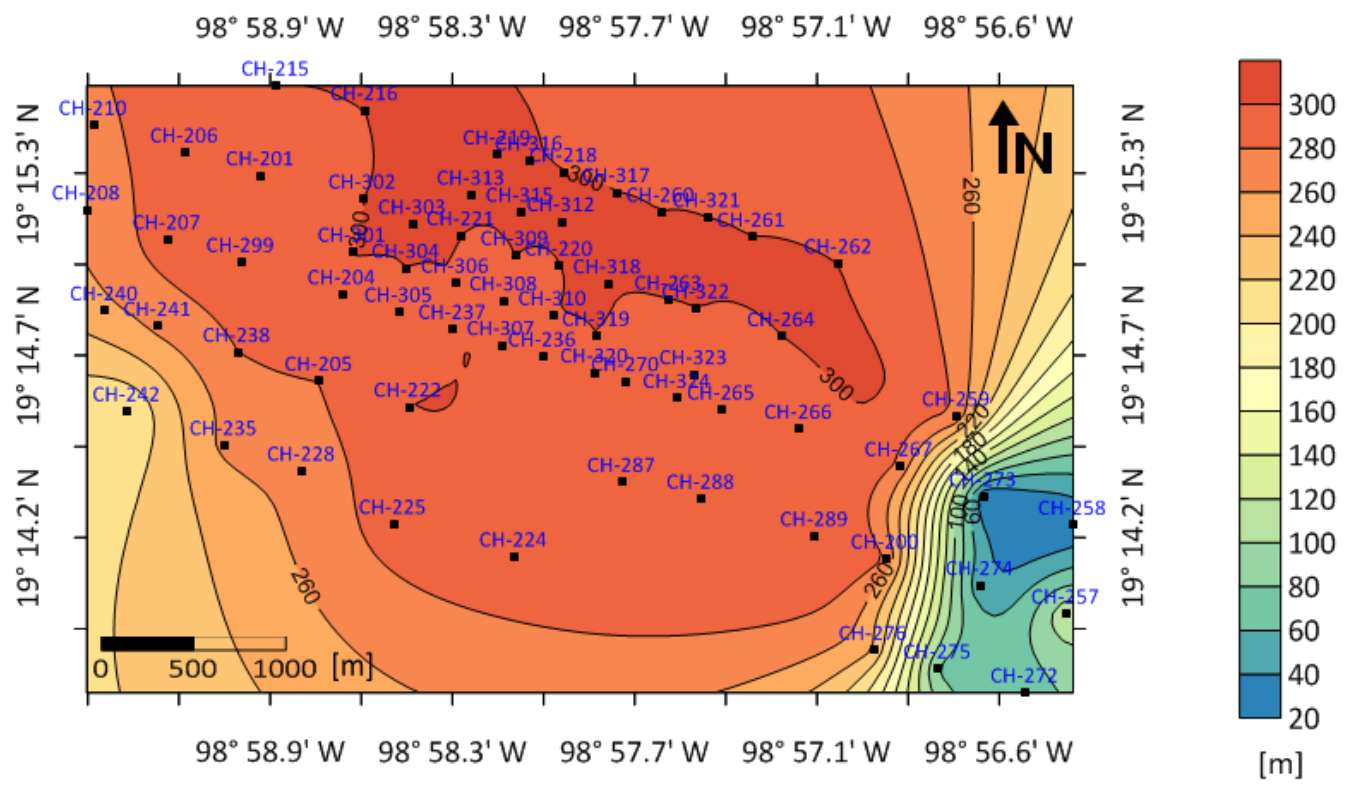

Figura 3 Mapa de distribución de espesores para el depósito lacustre, determinado a partir de la interpretación de los espectros H/V, y la identificaron de frecuencias dominantes, tomado de Vergara-Huerta, 2015.
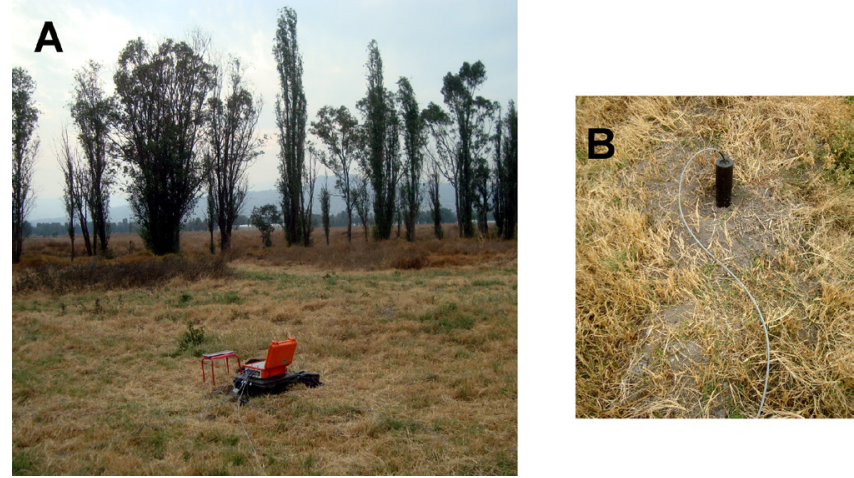

Figura 4 A) Medición de la variación del campo eléctrico y magnético para el sondeo magnetotelúrico (MT) usando el equipo ADU-07e (Metronix GmbH). B) Bobina de inducción de banda ancha para la medición del componente vertical del campo magnético.

minados. Un segundo aumento de la resistividad a profundidades entre 300 y $400 \mathrm{~m}$ corresponde con otro cambio importante en la composición del sedimento, asociado con el límite inferior de los depósitos de sedimentos lacustres y la presencia de depósitos volcaniclásticos y coladas de basalto. Los contactos inferidos del estudio geofísico ayudaron en la toma de decisiones sobre las herramientas para la perforación y fueron corroborados durante la perforación.

\section{Perforación}

$\mathrm{El}$ sitio de perforación seleccionado $\left(19^{\circ} 15^{\prime} 26^{\prime \prime} \mathrm{N}\right.$, 98 58'32"W) (Figura 1C-D) se encuentra dentro de la zona que de acuerdo con los datos geofisicos representó el depocentro de la cuenca de Chalco. El sitio es actualmente parte del ejido de Tulyehualco, y se localiza al SW del nuevo cuerpo lacustre que se ha formado por subsidencia, a un costado de la línea de pozos del ramal Mixquic-Santa Catarina (Figura 1D).

La perforación fue realizada por la compañía Major Drilling de México, empleando sistemas de perforación HQ y NQ con broca de diamante (Figura 5A y 5B). El tipo HQ fue utilizado para recuperar los sedimentos lacustres, relativamente suaves; esta herramienta perfora un pozo de 96.1 mm de diámetro y obtiene núcleos de sedimento de $63.5 \mathrm{~mm}$ de diámetro y aproximadamente 1.5 
$m$ de largo, que fueron recuperados dentro de en una camisa de plástico (figuras 5C y 5D). La herramienta NQ fue utilizada para perforar el material más resistente (rocas y materiales volcaniclásticos), esta herramienta perfora pozos de $75.7 \mathrm{~mm}$ de diámetro y obtiene núcleos de roca de $47.6 \mathrm{~mm}$ de diámetro y aproximadamente $1.5 \mathrm{~m}$ de largo, los cuales se recuperan en una caña de metal y se traspasaron a cañas de plástico del mismo diámetro. Durante la perforación se utilizaron bentonita en polvo y polímero biodegradable de celulosa, para aumentar el desempeño del fluido de perforación (estabilización del pozo, lavado de recortes, sellado de las fracturas de pozo, etc.). Las operaciones de perforación iniciaron el 23 de febrero y terminaron el 30 de marzo de 2016. Durante este tiempo se perforó de manera continua ( 24 horas $/ 7$ días a la semana).

Con el fin de obtener la secuencia sedimentaria lacustre lo más completa posible, fueron perforados tres pozos con traslape en las secciones longitudinales colectadas para posteriormente correlacionar las tres secuencias y construir una secuencia maestra continua. De esta manera se colectaron tres secuencias sedimentarias paralelas: secuencia A, de 10 a $420 \mathrm{~m}$; secuencia B de 6 a $310 \mathrm{~m}$ y secuencia $\mathrm{C}$ de 10 a $320 \mathrm{~m}$ y de 409 a $520 \mathrm{~m}$. Debido a que en ninguno de los tres pozos (A, B y $\mathrm{C}$ ) perforados por Major Drilling se recuperaron los

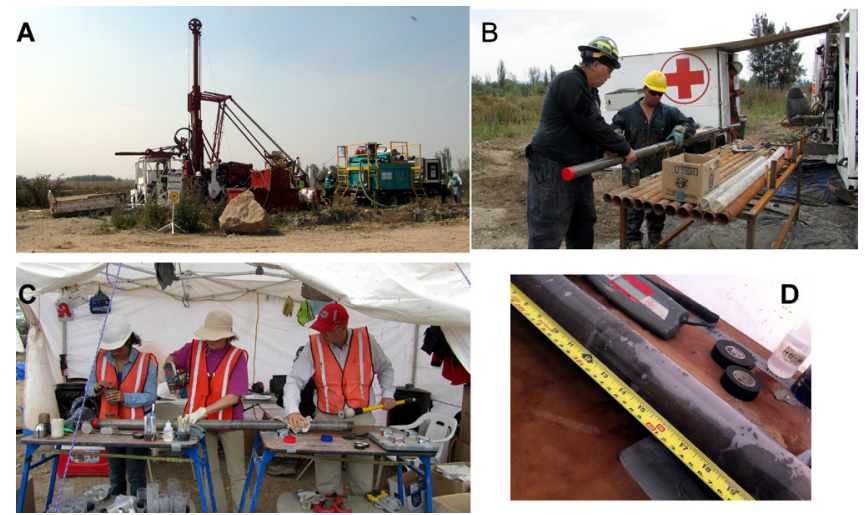

Figura 5 A) Torre de perforación, tuberías y separador de lodos. B) Extracción del tubo de plástico de la herramienta HQ. C) y D) Cada tubo de plástico fue limpiado, etiquetado y posteriormente pesado. sedimentos más superficiales, entre el 31 de marzo y el 2 de abril se perforó un cuarto pozo (secuencia D) empleando un equipo de perforación de pistón con motor de percusión con el que se recuperaron los primeros $17 \mathrm{~m}$ de la secuencia. De esta manera durante la campaña de perforación se recuperaron un total de $1152 \mathrm{~m}$ de sedimento, y se llegó a una profundidad máxima de $520 \mathrm{~m}$.

Durante la perforación de la secuencia A se realizó la documentación geofísica del pozo para obtener información sobre las propiedades físicas verticales de los sedimentos, a través de los registros de susceptibilidad magnética, resistividad eléctrica, espectro de rayos gamma y velocidad de ondas $\mathrm{P}$ (figuras 6A, 6B).

Además, durante la perforación del pozo B fue realizado un muestreo especial para estudios geomicrobiológicos y de metagenómica para lo cual se usó un trazador fluorescente que se añadió al fluido de perforación con el objetivo de controlar el nivel de infiltración del fluido de perforación en las muestras. Las muestras fueron colectadas de los primeros $100 \mathrm{~m}$ de la secuencia de este pozo y en cada tramo se tomaron muestras del fluido de perforación y del sedimento de la parte central y periférica de la zapata al momento en que los núcleos eran recibidos por el equipo científico. Para los análisis de metagenómica se colectaron alícuotas de $2 \mathrm{~cm}^{3}$ que fueron transferidas a bolsas de plástico y refrigeradas en una hielera y trasladadas al Instituto de Geología donde se congelaron a $-80{ }^{\circ} \mathrm{C}$.

Durante la perforación de todos los pozos, los núcleos recuperados y documentados en el sitio de perforación diariamente, y fueron trasladados al Instituto de Geología, de la UNAM, donde todas las secciones fueron montadas en un equipo automatizado GEOTEK en el que se realizaron mediciones de susceptibilidad magnética a intervalos regulares de $4 \mathrm{~cm}$ con un sensor de anillo Bartington (MS2C) (Figura 6C). Las gráficas generadas con estos datos fueron muy importantes para las decisiones diarias durante la perforación de los pozos, ya que permitieron ubicar de manera general el tipo de sedimento (lacustre vs. volcánico) que 

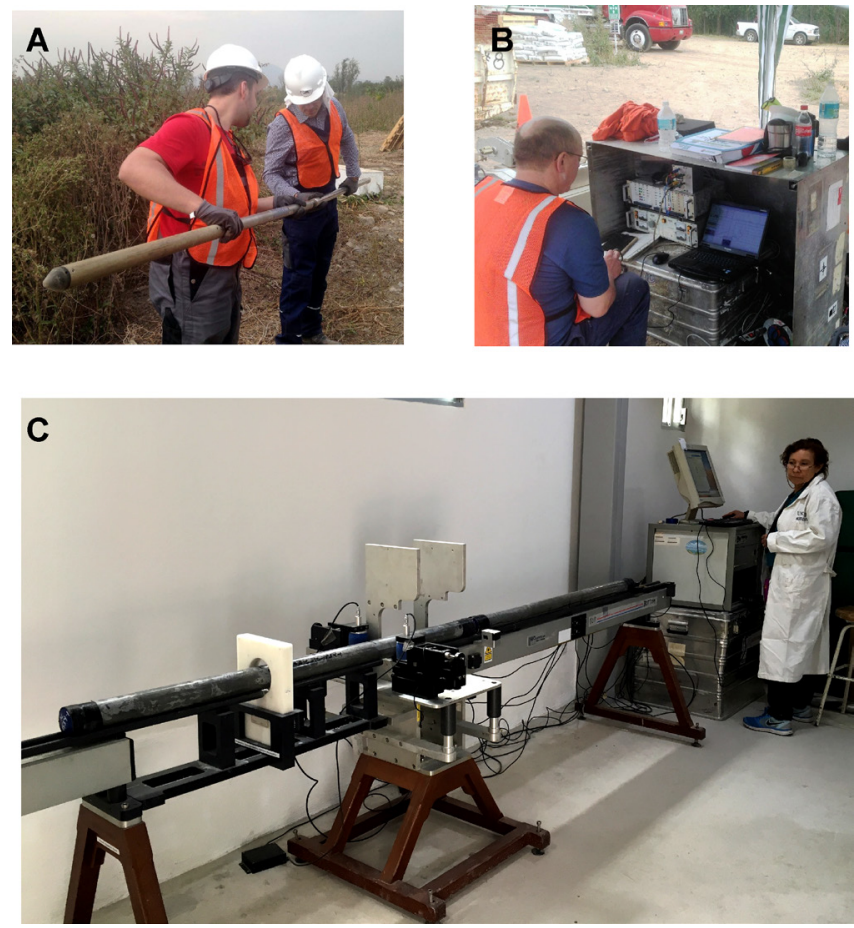

Figura 6 A) Sensor para registrar las variaciones de susceptibilidad magnética, resistividad eléctrica, espectro de rayos gamma y velocidad de ondas $p$ en el pozo $A$. B) Estación de registro para medición de los parámetros físicos. C) Equipo Geotek con anillo Bartington MS2C en el que se midió la susceptibilidad magnética en los núcleos recuperados.

se había atravesado y establecer correlaciones con los pozos anteriores.

En los laboratorios del Instituto de Geología también se realizaron preparaciones tipo frotis del material de la zapata de cada núcleo. Estas preparaciones se revisaron diariamente para identificar el tipo de sedimento que se estaba recuperando así como su contenido de microfósiles como diatomeas, ostrácodos, polen, etc.

\section{Resultados preliminares}

La recuperación de la secuencia sedimentaria de Chalco fue en general alta $(>85 \%)$, variando a lo largo de cada sondeo (Figura 7). En el sondeo 1A el porcentaje de recuperación global alcanzó el $88 \%$ obteniéndose la mejor recuperación entre los 30 a $80 \mathrm{~m}$ y de 310 a $420 \mathrm{~m}$ de profundidad.
En el sondeo B, el porcentaje de recuperación global fue de $91 \%$ con la mejor recuperación entre 30 a $90 \mathrm{~m}$. En el sondeo C se obtuvo un porcentaje de recuperación global del 92 \% con la mejor recuperación entre los 30 a los $260 \mathrm{~m}$. A partir de la correlación entre tres sondeos se construirá la secuencia maestra.

Se presentan ejemplos de algunas secciones de la secuencia sedimentaria (Figura 8A - 8F) donde según la textura del sedimento, fueron recuperadas arcillas limosas café con oozes de diatomeas con carbonatos masivos, sedimentos finamente laminados con oozes de diatomeas café claro y rojizos, sedimentos volcaniclásticos con cenizas y lapilli.

De acuerdo con los datos de susceptibilidad magnética (Figura 9) y con las observaciones realizadas en campo, aproximadamente los $260 \mathrm{~m}$ superiores de la secuencia están dominados por materiales clásticos de grano fino (Figura 8A - D), primordialmente de origen lacustre ricos en microfósiles, con valores relativamente bajos de susceptibilidad magnética, que están intercalados con horizontes de origen volcánico que se observan como máximos en la susceptibilidad magnética (Figura 8 F). Entre 260 y $300 \mathrm{~m}$ los valores de susceptibilidad magnética aumentan y los sedimentos están constituidos por material clástico más grueso intercalados con horizontes finos, posiblemente intercalaciones de depósitos de origen fluvial y lacustre. Por debajo de los $300 \mathrm{~m}$ los depósitos están constituidos por unidades volcaniclásticas con intercalaciones de flujos de lava y conglomerados aluviales, localmente alterados por hidrotermalismo entre 512 y $520 \mathrm{~m}$ de profundidad.

\section{Perspectivas}

Desde el punto de vista paleoclimático, la secuencia de Chalco, es un acervo único ya que puede contener la historia climática de varios ciclos glaciales - interglaciales. La información que se obtenga a través del estudio de la secuencia lacustre permitirá ayudar a probar varias hipótesis en re- 
Sondeo A

Sondeo B

Sondeo C
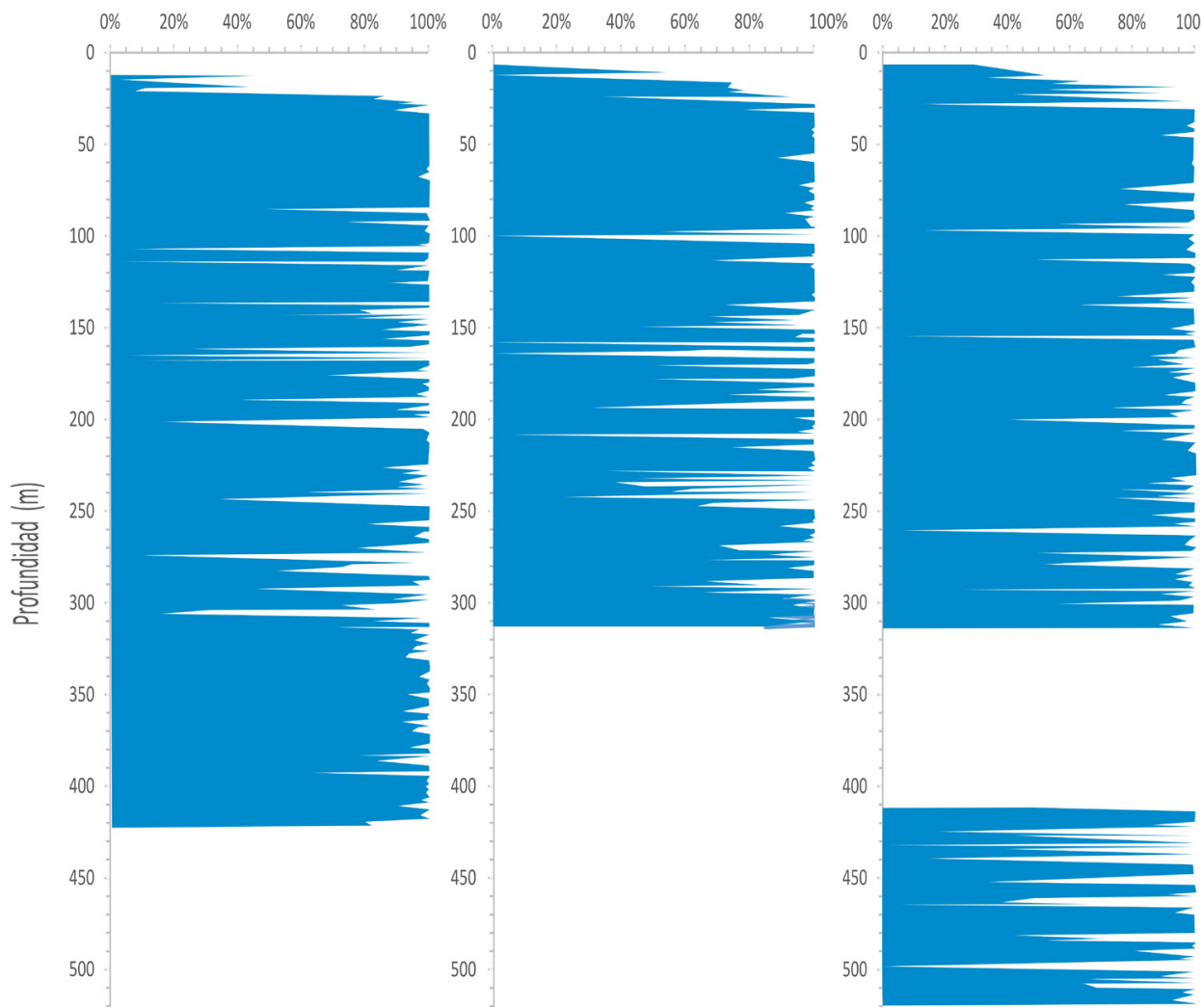

Figura 7 Porcentaje de recuperación de la secuencia de Chalco de las perforaciones de los sondeos A, B y C.

A

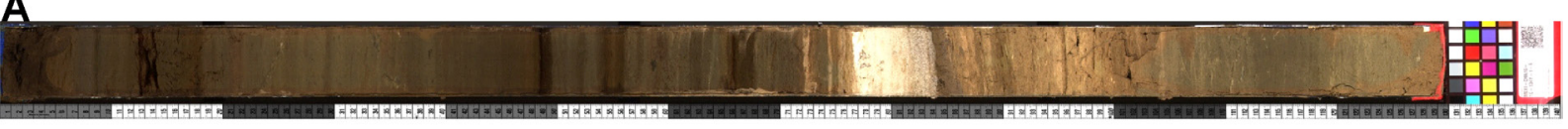

B

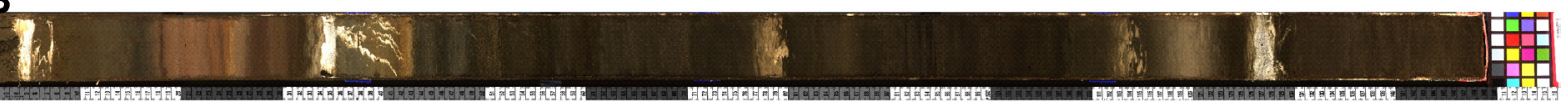

C

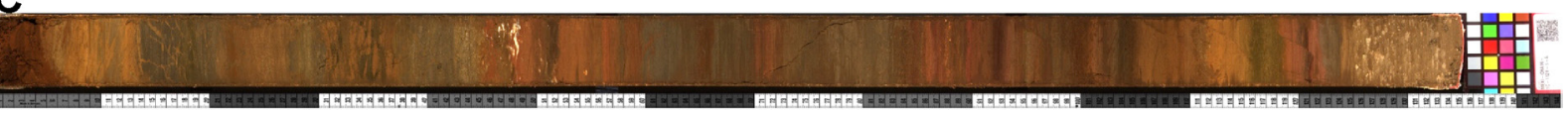

D

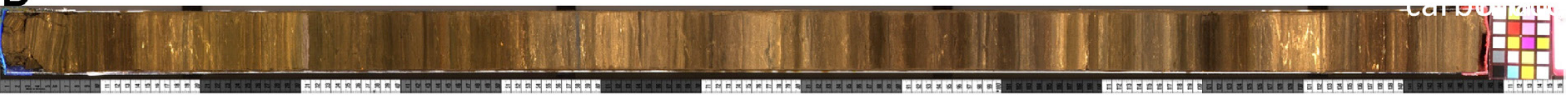

E

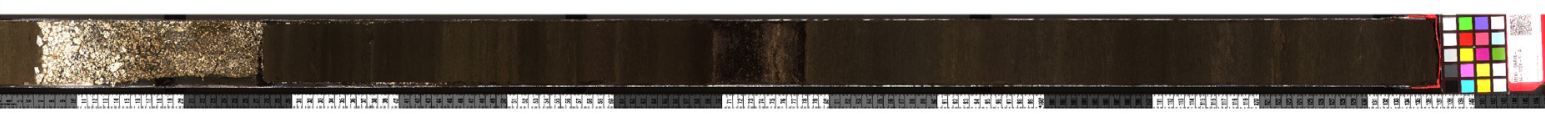

$\mathbf{F}$

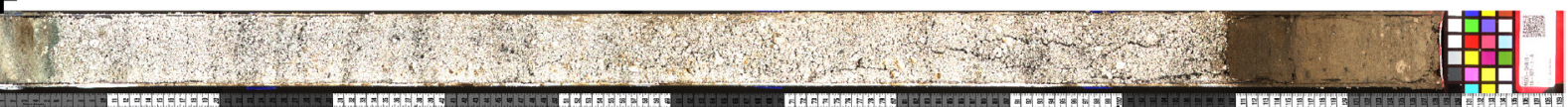

Figura 8 Secciones de la secuencia de Chalco. A) limos arcillosos, laminados, café obscuro y claro, con carbonatos. B) limos arcillosos, laminados y tefras claras. C) limos arcillosos, finos, rojizos, laminados. D) arcillas limosas finamente laminadas con oozes de diatomeas. E) limos arcillosos, negros, laminados, con tefras. F) lapilli. 


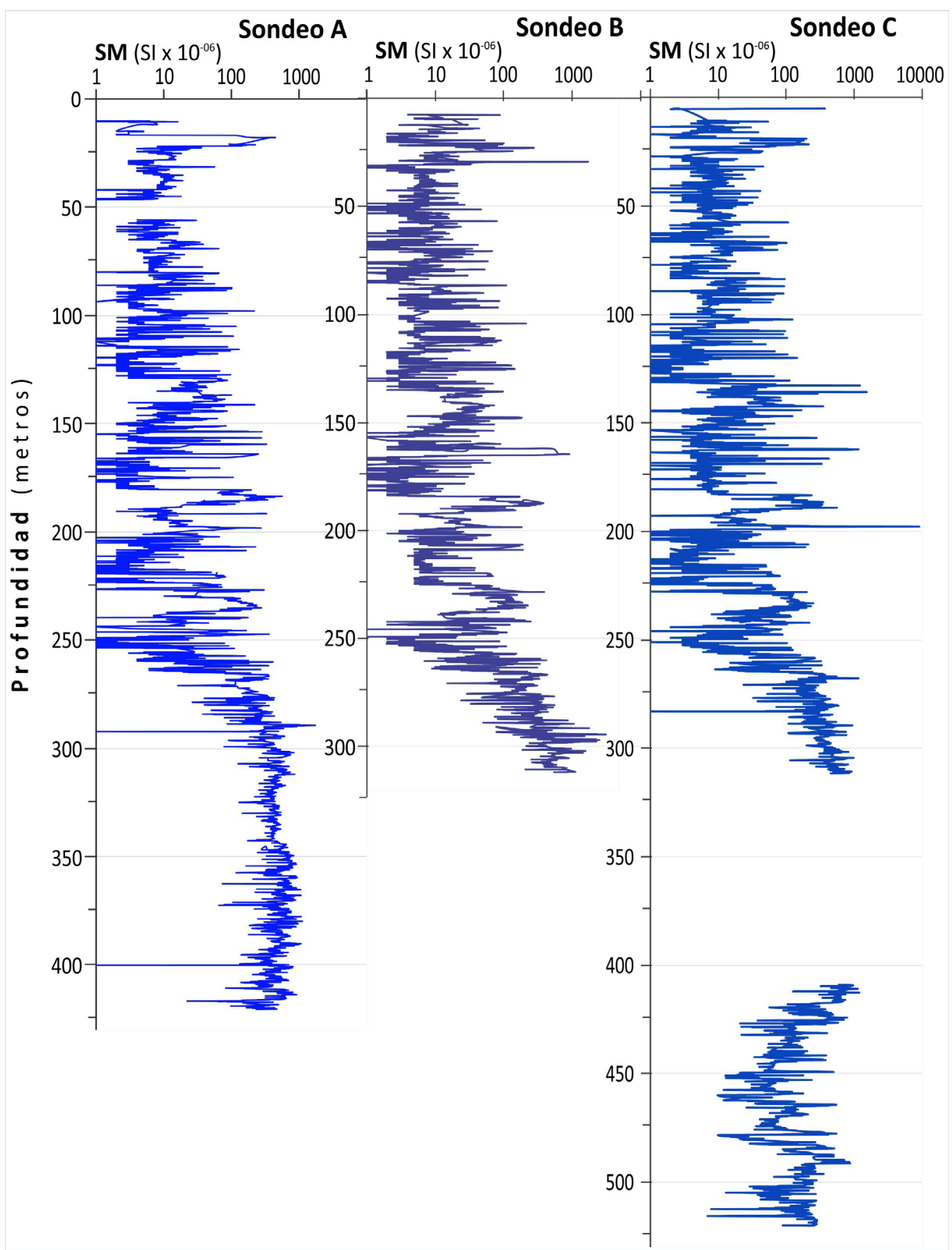

Figura 9 Registro de susceptibilidad magnética de los pozos A, B y C. Los datos se muestran en escala logarítmica.

lación al clima, la paleohidrología, y la dinámica de cambios abruptos durante los interglaciales y los glaciales. También permitirá aportar nuevas perspectivas sobre el comportamiento de los parámetros atmosféricos que controlan al clima de esta región, como son la intensidad del monzón mexicano y la posición latitudinal de la zona de convergencia intertropical.

Puesto que los sedimentos lacustres acumulados continuamente a través de miles de años en el lago de Chalco contienen indicadores geoquímicos, geológicos y ecológicos, a través de su análi- 
sis contaremos con información sobre el cambio ambiental inducido por los parámetros orbitales. En particular el análisis palinológico de los sedimentos permitirá reconocer las paleocomunidades de plantas que existieron y los cambios en su composición asociados a los cambios climáticos. Analizando el contenido de macropartículas de material carbonizado en los sedimentos, se podrán identificar los eventos de incendio. El estudio de las diatomeas, ostrácodos y otros fósiles preservados en estos sedimentos permitirá reconstruir la historia de este cuerpo lacustre, desde su formación hasta el momento actual, y permitirá identificar etapas de niveles lacustres más o menos profundos, asociados con climas más o menos húmedos dentro de los ciclos glaciales - interglaciales. Las variaciones en la composición geoquímica y mineralógica de los sedimentos permitirán reconocer los procesos de meteorización, erosión y diagénesis ocurridos durante la historia de su deposición. El conjunto de indicadores permitirán reconstruir los periodos de aridez y de humedad a lo largo de la secuencia sedimentaria.

El estudio de los componentes y las características geoquímicas de las tefras presentes en la secuencia permitirá identificar las potenciales fuentes de emisión, así como complementar el registro de la actividad volcánica y contribuir al establecimiento de los periodos de recurrencia para los distintos estilos de actividad eruptiva de la zona.

La comprensión de los mecanismos causantes del rápido cambio climático global en el pasado es crítico para la evaluación de los impactos del cambio climático actual y futuro. En particular, el papel de los trópicos en el cambio climático es un tema poco explorado y Chalco que es un sitio tropical de altura, podrá convertirse en el registro paleoclimático más importante de Norte América.

\section{Agradecimientos}

Este proyecto fue financiado por los siguientes proyectos: UNAM DGAPA-PAPITT IV100215,
International Scientific Drilling Program (ICDP Project 05-2014), US National Science Foundation (NSF-EAR1551311). Expresamos nuestro agradecimiento a la M. en G. Laura Luna por el apoyo en la elaboración del mapa del sitio. Agradecemos especialmente el invaluable apoyo de los ejidatarios del Ejido Santiago Tulyehualco gracias al cual se pudo realizar este trabajo, en particular queremos expresar nuestro reconocimiento al Sr. Gumesindo Jiménez Cabello, Sr. Juan Jiménez Xolapa y al Sr. Macedonio Jiménez Molotla.

\section{Referencias}

Arana-Salinas, L., Siebe, C., Macías, J.L., 2010, Dynamics of the $c a .4965 \mathrm{yr}{ }^{14} \mathrm{C}$ BP "Ochre Pumice" Plinian eruption of Popocatépetl volcano, México: Journal of Volcanology and Geothermal Research, 192(3), 212-231.

Arce, J.L., Layer, P.W., Morales-Casique E., Benowitz, J., Rangel, E., Escolero, O., 2013, New constraints on the subsurface geology of the Mexico City Basin: The San Lorenzo Tezonco deep well, on the basis of ${ }^{40} \mathrm{Ar} /{ }^{39} \mathrm{Ar}$ geochronology and whole-rock chemistry: Journal of Volcanology and Geothermal Research, 266, 34-49.

Bradbury, J.P., 1989, Late Quaternary lacustrine paleoenvironments in the Cuenca de Mexico: Quaternary Science Reviews, 8(1), 75-100.

Bücker, M., Lozano-García, S., Ortega Guerrero, B., Caballero Miranda, M., Pérez, L., Caballero-García, L., Pita de la Paz, C., Sánchez-Galindo, A., Villegas, FJ., Flores Orozco, A., Brown, E., Werne, J., Valero Garcés, B., Schwalb, A., Kemna, A., SánchezAlvaro, E., Launizar-Martínez, N., ValverdePlacencia, A., Garay-Jiménez, F., 2017, Geoelectrical and Electromagnetic Methods Applied to Paleolimnological Studies: Two Examples from Dried-Out Lakes of the Basin of Mexico: Boletín de la Sociedad Geológica Mexicana, 69(2), 279-298. 
Caballero, M., Ortega-Guerrero, B., 1998, Lake levels since about 40000 years ago at Lake Chalco, near Mexico City: Quaternary Research, 50, 69-79.

Cabral-Cano, E., Osmanoglu, B., Dixon, T., Wdowinski, S., DeMets, C., Cigna, F., DíazMolina, O., 2010, Subsidence and fault hazard maps using PSI and permanent GPS networks in central Mexico: International Association of Hydrological Sciences, Publication Series, 339, 255-259.

Campos-Enríquez, J., Delgado-Rodríguez, O., Chávez-Segura, R., Gómez-Contreras, P., Flores-Márquez, E., Birch, F., 1997, The subsurface structure of the Chalco sub-basin (Mexico City) inferred from geophysical data: Geophysics, 62, 23-35.

Gómez-Tuena, A., Orozco-Esquivel, M.T., Ferrari, L., 2005, Petrogénesis ígnea de la faja volcánica transmexicana. Boletín de la Sociedad Geológica Mexicana, 57(3), 227-283.

Guilbaud, M.N., Arana-Salinas, L., Siebe, C., Barba-Pingarrón, L.A., Ortiz, A. 2015, Volcanic stratigraphy of a high-altitude Mammuthus columbi (Tlacotenco, Sierra Chichinautzin), Central México: Bulletin of Volcanology, 77(3), 1-16.

Herrera-Hernández, D.A., 2011, Estratigrafía y análisis de facies de los sedimentos lacustres del Cuaternario tardío en la cuenca de Chalco, México, Posgrado en Ciencias de la Tierra, Universidad Nacional Autónoma de México (UNAM), Tesis de Maestría, 122p. Lozano-García, S., Ortega-Guerrero, B., Caballero-Miranda, M., UrrutiaFucugauchi, J., 1993, Late Pleistocene and Holocene paleoenvironments of the Chalco lake, Central Mexico: Quaternary Research, 40, 332-342.

Lozano-García, M.S., Ortega-Guerrero, B., 1994, Palynological and Magnetic Susceptibility Records of Chalco Lake, Central México:
Palaeogeography, Palaeoclimatology, Palaeoecology, 109,177-191.

Lozano-García, M.S., Ortega-Guerrero, B., 1998, Late Quaternary environmental changes of the central part of the Basin of Mexico; correlation between Texcoco and Chalco basins: Review of Paleobotany and Palynology, 99, 77-93.

Lozano-García, S., Ortega, B., Roy, P.D. Beramendi-Orosco, L., Caballero, M., 2015, Climatic variability in the northern part of the American tropics since the latest MIS 3: Quaternary Research, 85(2), 261-272.

Ortega-Guerrero, B., Thompson, R., UrrutiaFucugauchi, J., 2000, Magnetic properties of lake sediments from Lake Chalco, central Mexico, and their paleoenvironmental implications: Journal of Quaternary Science, 15(2), 127-140.

Ortega-Guerrero B., Lozano-García, S., Caballero, M., 2015, Historia de la evolución deposicional del lago de Chalco, México, desde el MIS 3: Boletín de la Sociedad Geológica Mexicana, 67(2), 185-201.

Pérez-Cruz, G., 1988, Estudio sismólogico de reflexión del subsuelo de la Ciudad de México, Universidad Nacional Autónoma de México (UNAM), Tesis de Maestría 83p.

Sánchez-Sesma, F.J., Rodríguez, M., IturraránViveros, U., Luzón, F., Campillo, M., Margerin, L., García-Jerez, A., Suárez, M., Santoyo, M., Rodríguez-Castellanos, A., 2011, A Theory for microtremor H/V Spectral ratio: Application for a layered médium: Geophysical Journal International, 186, 221-225.

Siebe, C., Arana-Salinas, L., Abrams, M, 2005, Geology and radiocarbon ages of Tláloc, Tlacotenco, Cuauhtzin, Hijo del Cuauhtzin, Teuhtli, and Ocusacayo monogenetic volcanoes in the central part of the Sierra Chichinautzin, México: Journal of Volcanology and Geothermal Research, 141 (3-4), 225-243. 
Torres-Rodríguez, E., Lozano-García, S., Roy, P., Ortega, B., Beramendi, L., CorreaMetrio, A., Caballero, M., 2015, Last Glacial droughts and fire regimes in the central Mexican highlands: Journal of Quaternary Science, 30(1), 88-99.

Urrutia-Fucugauchi, J., Lozano-García, M.S., Caballero-Miranda, M., Ortega-Guerrero, B., Böhnel, H., Hansen, R., Negendank, J.F.M., 1994, Paleomagnetic and Paleoenvironmental studies in the southern basin of Mexico. - I. Volcanosedimentary sequence and basin structure of Chalco Lake: Geofísica Internacional, 33, 421-430.
Vergara Huerta, F., 2015. Modelo de velocidad sísmica en la subcuenca de Chalco, Edo. de México, mediante análisis de cocientes $\mathrm{H} / \mathrm{V}$ de vibraciones ambientales. Posgrado en Ciencias de la Tierra, Universidad Nacional Autónoma de México (UNAM), Tesis Maestría, 96 p. 